\title{
Challenges to the application of IPMC as actuators of planetary mechanisms
}

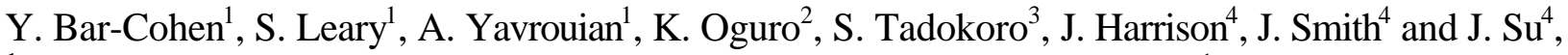 \\ JPL/Caltech, (MS 82-105), 4800 Oak Grove Drive, Pasadena, CA 91109-8099 ${ }^{1}$ \\ 2 Osaka National Research Institute, Osaka, Japan; \\ 3 Computer \& Systems Eng. Dept., Kobe University, Kobe, Japan; \\ 4 NASA Langley Research Center, Advanced Materials and Processing Branch, MS 226, Hampton, VA \\ 23681-2199
}

\begin{abstract}
Ion-exchange polymer membrane metallic composites (IPMC) are one of the electroactive polymers (EAP) that were shown to have potential application as actuators. The recent introduction of perfluorocarboxylategold composite with tetra-n-butylammonium and lithium cations instead of sodium made the most significant improvement of the material's electroactivity. Under less than 3 volts, such IPMC materials were shown to induce bending beyond a complete loop. The bending characteristic of IPMC offered an attractive actuation capability for a dust wiper in planetary applications and it was explored for the Nanorover's infrared camera window of the MUSES-CN mission. This joint NASA and the Japanese space agency mission, is scheduled to be launched from Kagoshima, Japan, in January 2002, to explore the surface of a small near-Earth asteroid. The application of EAP at space conditions posed a great challenge due to the harsh operating conditions that are expected and the critical need for robustness and durability. Several issues that are critical to the application of IPMC were addressed including operation in vacuum and low temperatures, as well as the effect of the electromechanical characteristics of the IPMC on its actuation capability. Highly efficient IPMC materials, mechanical modeling, unique elements and protective coatings were introduced to enhance the applicability of this EAP material. However, critical issues were identified that hamper the transition of IPMC from being considered for practical applications at this stage.
\end{abstract}

\section{INTRODUCTION}

Consideration of practical applications for electroactive polymers (EAP) has began only in this decade following the emergence of new materials that induce large displacements [Hunter and Lafontaine, 1992; Kornbluh, et al, 1995; and BarCohen, 1999a]. These materials are highly attractive for their low-density and large strain capability, which can be as high as two orders of magnitude greater than the striction-limited, rigid and fragile electroactive ceramics (EAC) [Bar-Cohen, et al, 1997; and Osada \& Gong, 1993]. Also, these materials are superior to shape memory alloys (SMA) in their temporal response, lower density, and resilience. However, EAP materials reach their elastic limit at low stress levels, with actuation stress that falls far shorter than EAC and SMA actuators. The most attractive feature of EAP materials is their ability to emulate biological muscles with high fracture tolerance, large actuation strain and inherent vibration damping. EAP actuation similarity to biological muscles gained them the name "Artificial Muscles" and potentially can be used to develop biologically inspired robots. The limited force actuation of current EAP is constraining the practical applications that can be considered.

Since EAP can be used as actuators that are light, compact and driven by low power, the authors sought to take advantage of their resilience and fracture toughness to develop space applications. The harsh environment associated with space poses great challenges to the application of EAP. Addressing these challenges has been the subject of the NASA task called Low Mass Muscle Actuators (LoMMAs). Under the lead of the principal author several EAP materials and applications were investigated. The emphasis of this paper is on Ion-exchange polymer membrane metallic composites (IPMC), which are bending EAP materials, first reported in 1992 [Oguro et al, 1992; Sadeghipour, et al, 1992 and Shahinpoor, 1992]. The various issues that can affect the application of IPMC were examined including operation in

\footnotetext{
${ }^{1}$ E-mail: yosi@jpl.nasa.gov; website: http://ndeaa.jpl.nasa.gov
} 
vacuum, low temperatures, and the effect of the electromechanical and ionic characteristics of IPMC on its actuation capability. The finding that IPMC can be activated at low temperatures and vacuum, paved the way for the serious consideration of this class of materials for space applications [Bar-Cohen, et al, 1997]. Its bending characteristics offered the potential to address the critical issue of planetary dust that affects solar cells and imaging instruments on such planets as Mars.

Throughout the authors' studies, several problem areas were identified as needing attention to assure the practicality of IPMC for application as actuators. The authors addressed these issues and the results of their study are reported herein.

\section{EAP ACTUATOR DRIVING DUST WIPER}

Lessons learned from the NASA Viking and Mars Pathfinder missions indicate that operation on Mars involves an environment that causes accumulation of dust on hardware surfaces. The dust accumulation is a serious concern that hampers long-term operation of optical instruments due to loss of visibility and degrades the efficiency of solar cells to produce power. To remove dust from surfaces one can use a similar mechanism as automobile windshield wipers. Contrary to conventional actuators, bending EAP has the ideal characteristics that are necessary to produce a simple, lightweight, low power wiper mechanism. Specifically, the IPMC responds to activation signals at a frequency of less than a Hertz with a bending angle that can exceed 90 degrees span each way depending on the polarity. Since an EAP wiper was demonstrated to remove dust effectively (see Figure 1a), the MUSES-CN mission selected it as a baseline technology for the Nanorover's infrared camera window. This mission is a joint effort of NASA and the NASDA (National Space Development Agency of Japan), which is scheduled to be launched from Kagoshima, Japan, in January 2002, to explore the surface of a small near-Earth asteroid.

An IPMC actuator drove the dust wiper, which was used for the technology demonstration (shown in Figure 1a). A blade with a fiberglass brush was attached to the actuator and it was used to mechanically remove dust particles that were sprinkled onto a glass plate. Since the expected dust particle size may be as small as fraction of microns efforts were made to enhance the wiping mechanism and an electric repulsion mechanism was introduced (see Figure 1b). For this purpose, the wiper was introduced with about $1.5 \mathrm{KV}$ using a battery driven circuit and the dust was repelled as the blade moves above the surface.

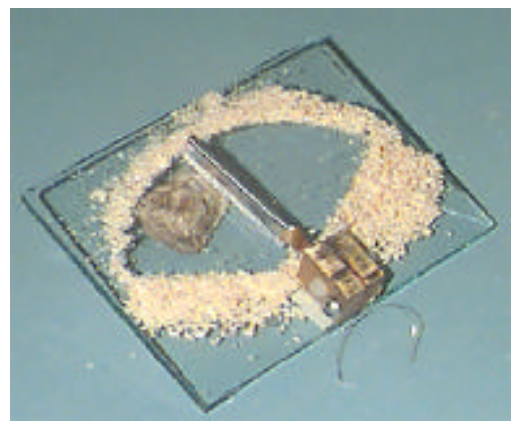

FIGURE 1a: IPMC dust wiper removing sawdust from a glass window

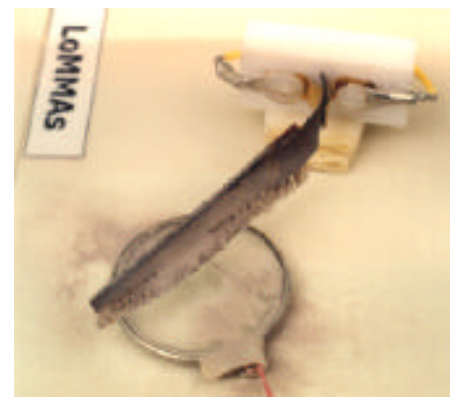

FIGURE 1b A wiper blade actuated by an IPMC actuator is subjected to high voltage to repel dust.

A unique 100-mg wiper blade was constructed by ESLI (San Diego, CA) using a composite (graphite fibers and DuPont Kapton $^{\mathrm{TM}}$ resin) beam with fiberglass brush (Figure 2). The blade was processed at a temperature of $623 \mathrm{~K}\left(350^{\circ} \mathrm{C}\right)$ and was tested to demonstrate endurance down to $77 \mathrm{~K}$. A $15-\mathrm{mm}$ x $6-\mathrm{mm}$ IPMC film was bonded to the ESLI blade using platinum electrode strips bonded on its other end to provide electrical excitation of the EAP wiper. Since mechanical wiping of a surface using a soft brush may not remove minute dust particles, which are smaller than the distance between the whiskers, a high voltage repulsion mechanism was introduced. The blade (beam and brush) was coated with gold and activated by a 1-2-KV bias DC voltage (with the metal ring around the glass piece, whereas the EAP wiper was subjected to $45 \mathrm{~V}$ (Figure 2). For dust cleaning from windows, it is necessary to place the wiper outside the viewing area and move it inward to clean the window. This necessitates the use of two wipers that are placed on opposite sides of the window as shown in Figure 3. In an effort to bring the technology to space flight readiness the critical issues associated with the IPMC material as an actuator were addressed.

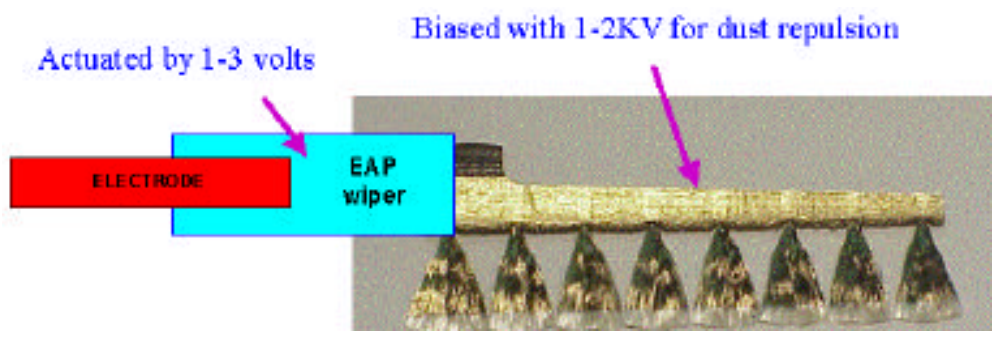


FIGURE 3: A schematic view showing the area that is covered by two bending EAPactuators sweeping a window from two opposite sides.

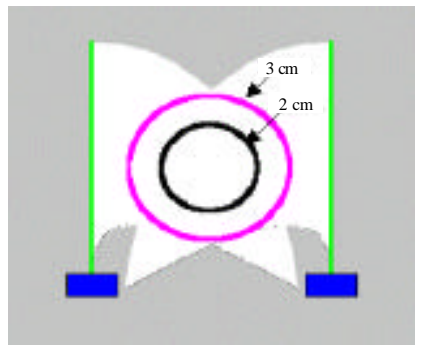

\section{MODELING THE ACTUATOR}

Design and prediction of the response of the IPMC dust wiper requires an effective analytical modeling of the material and its mechanical constraints. For this purpose, the Kanno-Tadokoro model was adopted using a gray box approach relating the experimental input and output data. The voltage applied to an actuator is transformed to current distribution through the membrane. The current generates distributed internal stress, which causes strain in the IPMC material and it is affected by its viscoelastic properties. The resistances of surface layers and RC elements approximate the experimental voltage-current response as the electric property. The stress generation property and the viscoelasticity were expressed by an equation similar to the piezoelectric equation.

$$
\sigma=D(s) \varepsilon-e i \frac{\omega_{n}^{2} s}{s^{2}+2 \varsigma \omega_{n} s+\omega_{n}^{2}}
$$

Where: $\sigma$ - internal stress; $D(s)$ - mechanical characteristics including mass, damping and stiffness; $\varepsilon$ - strain vector; $e$ transformation tensor of stress generation; $i$ - current through the actuator; and $\zeta$ and $\omega_{n}$-delay parameters of the $2^{\text {nd }}$ order.

The 2nd order delay approximates the time delay until ionic distribution reaches equilibrium and the internal stress is generated by swelling and electrostatic force. This equation was used to simulate the response of the IPMC actuators and in Figure 4a the results for 15-mm long 8-mm wide actuator strip in shown. The strain near the electrodes clamp (fixture) is larger than the tip. Analysis, using this model, has shows that current concentration near the electrodes causes imbalance of strain distribution. The response speed is faster near the electrodes because of the $\mathrm{RC}$ elements and therefore, it is better to design shorter actuator. The whole membrane deforms to curve in two dimensions, where deformation in the direction of width obstructs the wiper motion. When a $2 \mathrm{~mm}$ long section of the IPMC tip is constrained preventing it from deformation, the bending displacement is improved as shown in Figure $4 \mathrm{~b}$. Therefore, a crosspiece design is important for efficient actuation.
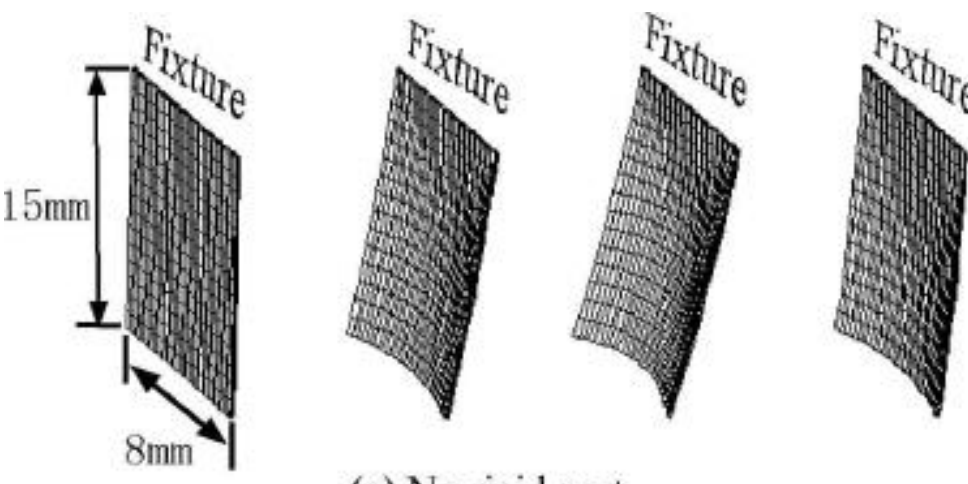

(a) No rigid part
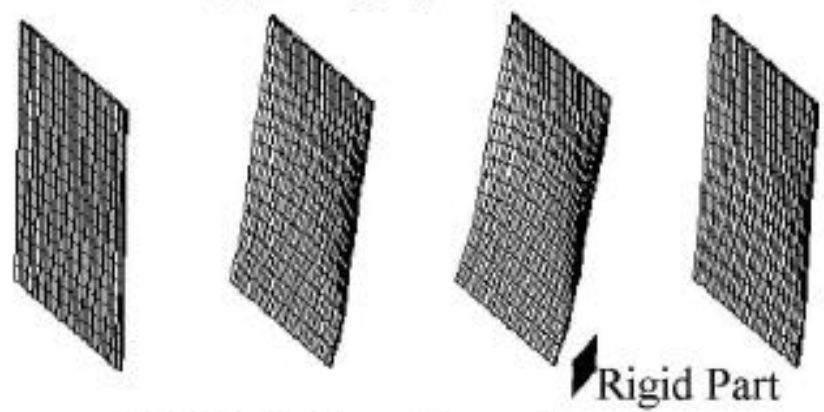

(b) With rigid part $2 \mathrm{~mm}$ long

FIGURE 4: Simulation result of actuator motion.

\section{IPMC AS A BENDING EAP ACTUATOR}

Driving a dust wiper using EAP materials requires that the material be capable of bending under electro-activation. As can be seen for the first two issues of the WW-EAP Newsletter [Bar-Cohen, 1999b], several types of EAP materials can be made to bend under electrical excitation. The authors concentrated on the use of the IPMC with metal electrodes deposited on both sides. Two types of base polymers were used: Nafion ${ }^{\circledR}$ (perfluorosulfonate made by DuPont) and Flemion ${ }^{\circledR}$ (perfluorocaboxylate made by Asahi Glass, Japan). Prior to using these polymers as EAP base material, they were widely employed in fuel cells and production of hydrogen (electrolysis) [Holze and Ahn, 1992]. The operation as actuators is the reverse process of the charge storage mechanism associated with fuel cells. In the current study, 
Nafion ${ }^{\circledR} \# 117$ was used with a thickness of $0.18-\mathrm{mm}$ and perfluorocarboxylate films were used having a thickness of 0.14 $\mathrm{mm}$. Initial studies involved the use of platinum as the metal electrode however recent studies have shown that a gold coating provides superior performance [Abe, et al, 1998]. The gold layer was applied in 7-cycles resulting in a dendritic structure as shown in a cross section view in Figure 5. The counter cation consists of tetra-n-butylammonium or lithium and these two species showed significantly greater bending response than sodium, which was used earlier. Under less than $3-\mathrm{V}$, such IPMC materials were shown to bend beyond a complete loop and the response follows the electric field polarity.

FIGURE 5: Perfluorocarboxylate membrane with tetra-n-butylammonium cation and 7 cycles of ion exchange and reduction (resulting dendritic growth) of the gold electrodes

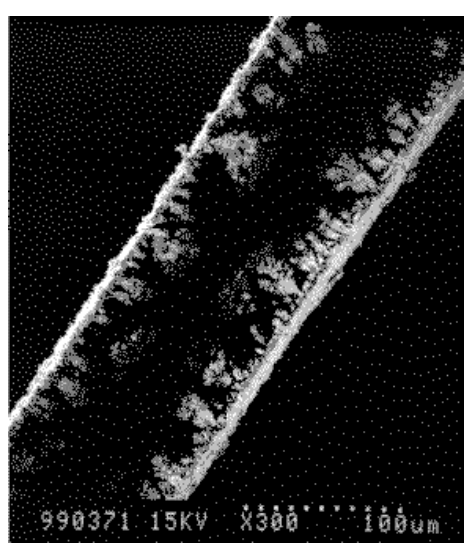

When an external voltage is applied on an IPMC film, it causes bending towards the anode at a level that increases with the voltage, up until reaching saturation, as shown in Figure 6. Under AC voltage, the film undergoes swinging movement and the displacement level depends not only on the voltage magnitude but also on the frequency. Generally, activation at lower frequencies (down to 0.1 or $0.01 \mathrm{~Hz}$ ) induces higher displacement and the displacement diminishes as the frequency rises to several tens of Hz. The drive voltage level at which the bending displacement reaches saturation depends on the frequency and is smaller at higher frequencies. The applied electrical current controls the movement of the film but the response is strongly affected by the water content of the IPMC serving as an ion transport medium.

FIGURE 6: The response of the bending EAP to various voltage amplitudes at three different frequencies (data obtained for sodium base IPMC).

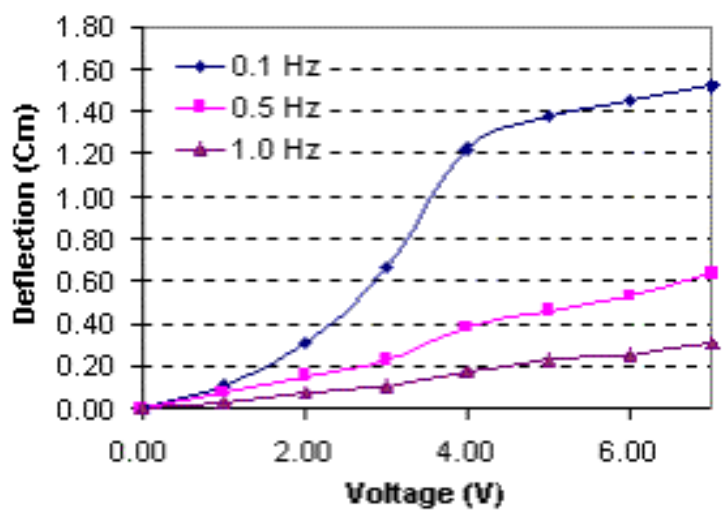

The authors addressed several issues that were determined critical to the application of IPMC (both the Nafion ${ }^{\circledR}$ and Flamion ${ }^{\circ}$ base):

Film moisture: IPMC is highly sensitive to hydration. To maintain the moisture content there is a need for a protective coating, otherwise the material stops to respond after a few minutes of activation in dry conditions. Using an etching procedure and silicone coating, an IPMC film was shown to operate for about 4 months. This Dow Corning coating material allows operation in a wide range of temperatures with great flexibility and is durable under UV radiation. However, since the MUSES-CN mission requires operation over 3-years, the 4-month protection period is too short. Analysis of the cause of the degradation indicated that the silicone coating is water permeable and the rate is $3000 \mathrm{~cm}^{3} x$

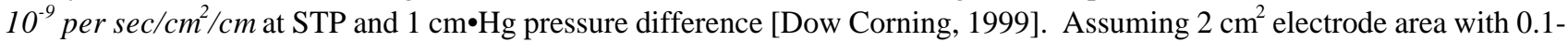
$\mathrm{mm}$ thick silicone, the coating shows a water loss rate of $\sim 40-50 \mathrm{mg} / 24 \mathrm{hrs}$. This rate is significantly higher than observed for IMPC and does not account for the IPMC electrode layers, however it indicates the severity of the issue. To overcome this limitation various alternative coating techniques are being considered including the use of a metallic selfassembled monolayer as an overcoat.

Electrolysis: The wetness of IPMC and the introduction of voltages at levels above 1.23-V introduce electrolysis during electro-activation causing degradation, heat and release of gasses. This issue raises a great concern since the emitted hydrogen and oxygen accumulate under the protective coating and leads to blistering, which will rupture the coating due to the high vacuum environment of space. The use of tetra-n-butylammonium cations was shown to provide higher actuation efficiency allowing to reduce the needed voltage and to minimizing the electrolysis effect. 
Operation in vacuum and low temperatures: In space the temperature can drop to significantly low levels and the ambient pressure is effectively vacuum. The ability to protect IPMC from drying allowed performing tests in vacuum and low temperatures. These tests showed that while the response decreases with temperature, as shown in Figure 7, a sizeable displacement was still observed at $-140^{\circ} \mathrm{C}$. This decrease can be compensated by an increase in voltage. It is interesting to point out that, at low temperatures, the response reaches saturation at much higher voltage levels than room temperatures.

FIGURE 7: Deflection amplitude of sodium-base IPMC as a function of time and temperature.



Besides the need to address the low temperature issue, the material ability to sustain temperatures as high as $+125^{\circ} \mathrm{C}$ is also necessary. For this purpose, several solvents with higher boiling points than water were examined for their potential use as a solvent for the IPMCs. These solvents were examined for their performance equivalence to "antifreeze" in automobile radiators. Various solvents were considered and their effect on the swelling characteristics of Nafion ${ }^{\circledR}$ was investigated. Nafion ${ }^{\circledR}$ strips with an initial size of $5.8 \mathrm{~mm} \times 38.1 \mathrm{~mm}$ were immersed in a series of solvents for a period of 4days at ambient temperature and the change in mass and size were measure. The results are listed in Table 1 and show both swelling and increase in mass due to water absorption. Examination of IPMC films that were immersed for 24-hours in various solvents, including ethylene glycol, showed a significant reduction in the induced bending amplitude.

TABLE 1: Changes in mass and size as a result of immersing Nafion® in various liquid media for 4-days at ambient temperature.

\begin{tabular}{|l|c|c|c|c|}
\hline \multicolumn{1}{|c|}{ Solvent } & $\begin{array}{c}\text { Initial mass, } \\
\mathrm{g}\end{array}$ & Final mass, g & Change, \% & $\begin{array}{c}\text { Dimensions after soaking } \\
\text { [width } \mathrm{x} \text { length }(\mathrm{mm})\end{array}$ \\
\hline Water & 0.0722 & 0.0838 & 16 & $5.8 \times 43.0$ \\
\hline $\begin{array}{l}\text { N-methyl-2-pyrrolidinone } \\
\text { (NMP) }\end{array}$ & 0.0721 & 0.1124 & 56 & $6.4 \times 50.8$ \\
\hline Ethanol & 0.0707 & 0.1113 & 57 & $6.4 \times 50.8$ \\
\hline Dimethylformamide (DMF) & 0.0606 & 0.0974 & 61 & $6.4 \times 44.5$ \\
\hline Ethylene glycol & 0.0720 & 0.1104 & 53 & $6.4 \times 50.8$ \\
\hline Ammonium hydroxide & 0.0719 & 0.0795 & 11 & $5.8 \times 41.4$ \\
\hline
\end{tabular}

TABLE 2: Challenges and identified solutions for issues regarding the application of IPMC.

\begin{tabular}{|l|l|}
\hline \multicolumn{1}{|c|}{ Challenge } & \multicolumn{1}{c|}{ Solution } \\
\hline Fluorinate base - difficult to bond & Apply pre-etching \\
\hline Sensitive to dehydration & Apply protective coating over pre-etched IPMC \\
\hline Off-axis bending actuation & Constrain the free end \\
\hline Operate at low/high temperatures & IPMC was demonstrated to operate at - $140^{\circ} \mathrm{C}$ \\
\hline Remove small size dust & Use effective wiper-blade design and high bias voltage \\
\hline Protective coating is permeable & Develop alternative coating possibly using overcoat \\
\hline Low actuation force & Needs enhancement of the material performance \\
\hline Complex equivalent circuit characteristics & Needs improved understanding \\
\hline Reverse bending under DC voltage & Limit application to dynamic operations \\
\hline $\begin{array}{l}\text { Electrolysis occurs at }>1.23-V \text { causing blisters } \\
\text { under the coating }\end{array}$ & Use efficient IPMC requiring lower actuation voltage \\
\hline No established quality assurance & Use short beam/film and tackle the critical issues \\
\hline Residual deformation & Still a challenge \\
\hline
\end{tabular}


Low actuation force: Using thin IPMC with a thickness of $0.14-\mathrm{mm}$ was found to induce a significant bending displacement. However, the induced force was found to be relatively small making it difficult for the wiper to overcome the electrical forces that are involved with the dust repelling high-voltage. Further, even though the wiper blade is relatively light, weighing about 104-mg, it is still responsible for significant bending due to gravity pressing the blade onto the window surface and constraining its movement. Alternative $0.18-\mathrm{mm}$ thick film is currently being sought to provide the necessary force.

Permanent deformation under DC activation: Unfortunately, under DC voltage IPMC strips do not maintain the actuation displacement and retract after several seconds. Further, upon removal of the electric field an overshoot displacement occurs in the opposite direction moving slowly towards the steady state position leaving a permanent deformation. This issue was not resolved yet and would hamper the application of IPMC.

Challenges and solutions: To allow future design of EAP mechanisms actuated by IPMC, the challenges and solutions were summarized and are listed in Table 2. While most challenges seem to have been addressed, two issues still pose a concern: the introduction of permanent deformation and the need for an effective protective coating. Unless these issues are effectively resolved the use of IPMC for planetary applications will be hampered.

\section{CONCLUSION}

In recent years, electroactive polymers have emerged as actuators with great potential to enable unique mechanisms that can emulate biological systems. A study has taken place to adapt IPMC as an actuator of a dust wiper for a mission to an asteroid. A series of challenges were identified as obstacles to the transition of such materials to space flight missions. Some of the key issues were effectively addressed while some require further studies. The unresolved issues include a permanent deformation under DC activation and the water permeability of the developed protective coating. Another issue is the limited force that is induced by IPMC, which require compromising between the bending displacement and the actuation capability. The unresolved challenges identified in this study are recognized as critical to the potential transition of IPMC to practical applications. Currently, alternative EAP materials are being considered.

\section{ACKNOWLEDGMENT}

The research at Jet Propulsion Laboratory (JPL), California Institute of Technology, was carried out under a contract with National Aeronautics and Space Agency (NASA) Code S, as part of the Surface Systems Thrust area of CETDP, Program Manager Dr. Samad Hayati.

\section{REFERENCES}

Abe, Y., A. Mochizuki, T. Kawashima, S. Yamashita, K. Asaka and K. Oguro"."Effect on Bending Behavior of Counter Cation Species in Perfluorinated Sulfonate Memberance-Platinum Composite," Poly. for Adv. Tech., Vol. 9 (1998), pp. 520-526.

Bar-Cohen Y. (Ed.), WorldWide Electroactive (WW-EAP) Newsletter, Vol. 1, No. 1, http//ndeaa.jpl.nasa.gov/nasande/lommas/eap/WW-EAP-Newsletter.html (1999b)

Bar-Cohen, Y., (Ed.), Proceedings of the Electroactive Polymer Actuators and Devices, Smart Structures and Materials 1999, Volume 3669, pp. 1-414, (1999a).

Bar-Cohen, Y., S. Leary, M. Shahinpoor, J. Harrison and J. Smith, "Flexible Low-Mass Devices and Mechanisms Actuated by Electroactive Polymers," ibid, pp. 51-56 (1999).

Dow Corning data for general silicones - based on personal communication, Oct. 1999.

Heitner-Wirguin C., "Recent advances in perfluorinated ionomer membranes: Structure, properties and applications," J. of Membrane Sci., V 120, No. 1, 1996, pp. 1-33.

Holze R., and J. C. Ahn, "Advances in the use of Perfluorinated Cation-Exchange Membranes in Integrated Water Electrolysis and Hydrogen - Oxygen Fuel Systems," J. Membrane Sci., Vol. 73, No. 1, pp. 87-97 (1992).

Hunter I. W., and S. Lafontaine, "A comparison of muscle with artificial actuators," IEEE Solid-State Sensor and Actuator Workshop, 1992, pp. 178-165.

Kornbluh R., R. Pelrine and Joseph, J. "Elastomeric dielectric artificial muscle actuators for small robots," Proceeding of the $3^{\text {rd }}$ IASTED Intern. Conf., Concun, Mexico, June, 14-16, 1995.

Oguro, K., Y. Kawami and H. Takenaka, "Bending of an Ion-Conducting Polymer Film-Electrode Composite by an Electric Stimulus at Low Voltage, ” Trans. J. of Micromachine Soc. , 5, (1992) 27-30.

Osada, Y. and J. Gong, "Stimuli-Responsive Polymer Gels and Their Application to Chemomechanical Systems," Prog. Polym. Sci, 18 (1993) 187-226.

Sadeghipour, K., R. Salomon, and S. Neogi, "Development of a Novel Electrochemically Active Membrane and 'Smart' Material Based Vibration Sensor/Damper,” Smart Mater. and Struct., (1992) 172-179.

Shahinpoor, M., "Conceptual Design, Kinematics and Dynamics of Swimming Robotic Structures using Ionic Polymeric Gel Muscles," Smart Mater. and Struct., Vol. 1, No. 1 (1992) 91-94. 